\title{
Pressure Variation in a Fluid Flow over Non-Uniform, Porous Bottom Topography
}

\author{
Godwin Christopher Ezike Mbah, Emmanuel Oluwakorede Oshilim \\ Department of Mathematics, University of Nigeria, Nsukka, Nigeria \\ Email:godwin.mbah@unn.edu.ng,emmanuel.oshilim.pg03561@unn.edu.ng
}

How to cite this paper: Mbah, G.C.E. and Oshilim, E.O. (2020) Pressure Variation in a Fluid Flow over Non-Uniform, Porous Bottom Topography. Open Access Library Journal, 7: e6033.

https://doi.org/10.4236/oalib.1106033

Received: December 27, 2019

Accepted: February 25, 2020

Published: February 28, 2020

Copyright $\odot 2020$ by author(s) and Open Access Library Inc.

This work is licensed under the Creative Commons Attribution International License (CC BY 4.0).

http://creativecommons.org/licenses/by/4.0/

(c) (i) Open Access

\begin{abstract}
This paper presents pressure variation in fluid flow over a porous media. In the model, we considered water as an incompressible fluid: the flow as nonsteady and uniform. We derived an equation for the nonuniform bottom topography (flow depth) and substituted into the governing equation for shallow water flow with nonuniform bottom topography. We made use of Darcy's law to construct equation for Darcy flux, which in turn related pressure gradient to the flow velocity, the porosity, and the permeability of the porous media. From the governing equation of shallow water flow with nonuniform bottom topography, we solved for the flow velocity using Homotopy Perturbation Method (HPM). We incorporated the flow velocity into the equation for the pressure gradient and solved for the pressure variation in the channel. We analyzed and found out that, the higher the permeability the lower the pressure within the flow and the lower the permeability the higher the pressure, because there is going to be a pressure build-up under this condition. We also found that the higher the flow height $(H)$ the higher the pressure.
\end{abstract}

\section{Subject Areas}

Fluid Mechanics

\section{Keywords}

Pressure Variation, Porous Media, Darcy’s Law, Porosity, Permeability

\section{Introduction}

The modern understanding of fluid motion began several centuries ago, with the work of L. Euler, Isaac Newton, Bernoulli, Lagrange etc. [1]. The ocean and the atmosphere remain phenomenal and have been a wonder, source of curiosity over the years. It's properties like buoyancy, wave and weather, transport of 
mass and heat, all have been a source of curiosity to the scientist and the philosopher over the years till date [2]. One of their contemplations is to understand why the ocean and the atmosphere behave as they do. The major forces that influence flow in open channel are those of inertia, gravity and pressure gradient [3]. The study of shallow water flow is important, for it can be used to predict the insurgent of natural phenomena like tsunami, flooding, rise in water level etc. One of the motivations to study shallow water over a porous non-uniform bottom topography is to understand the effect of porous medium on the water flowing in the channel.

Porous medium is a solid containing void spaces (spores) either connected or unconnected, dispersed within it in either regular or random manner. If the pore represents a certain portion of the bulk volume, a complex network can be formed, which is able to carry fluid or even store it [4].

[5] worked on shallow water wave and assumed a slopping bottom topography where he treated the wave to be linear. He considered the effect of dispersion on the flow mechanism, which many work before his did not consider. Later on, [6] considered the flow to have a constant flow depth, which of course is not realistic enough. [7] also contributed in this area, by taking into consideration non-uniformity of bottom topography at different points. Their work is close to reality but for their assumption that angular measurement of the non-uniform bottom region is constant cannot be taken as true.

[8] extended the work by [7], by taking the angular measurement of the depth of the non-uniform bottom region to be changing with respect to time. A typical case, he pointed out is the erosion flow over a contoured region along the flow path, like sandy area, where the non-uniform region is being filled by the debris transported by the flow. This is more realistic than [7], but this work does not completely describe the dynamics of non-uniform bottom topography of a natural channel. [9] investigated transcritical shallow water flow past topography. In their work they considered an obstacle or say ridge on the bed of the channel. They considered upstream and downstream waves that arise due to the obstacle that localized in the flow direction. They used the framework of the forced Su-Gardner (SG) system of equation. They took a primary focus on the transcritical regime when the Froude number of the oncoming flow is close to unity. [3] modeled an open channel flow over porous media (river). They considered water as incompressible fluid and assumed a steady and uniform flow, laminar flow pattern and the system to be isothermal. They used an analytical method to solve the resulting Brinkman equation. They found out that flow velocity decreases with an increase in permeability and increases in the height of the channel increased flow velocity. Also, that increase in permeability resulted in a decrease in pressure. [10] studied shallow water flow over non-uniform varying bottom topography, which is an extension from work by [6] and [8], they employed the use of perturbation method to solve the governing equation for shallow water flow over non-uniform varying bottom topography. Their solution to 
the wave equation for shallow water flow showed there exists a single wave elevation which still has a singularity in the profile.

This work stemmed from the work of [3] [8] and [10] and we are going to look into a situation where such contoured region is being dug continually, what happens over time if it is not controlled and effect of permeability on pressure gradient along the channel.

\section{Model Formulation}

We see that the total depth of the flow from the free surface is given by:

$$
\begin{gathered}
H=h+y^{\prime}+\delta y \\
\Rightarrow H=h+x \cot \beta
\end{gathered}
$$

from Figure 1, Equation (1) above gives the flow depth of the contour region. We recall Darcy's law:

$$
Q=-k \frac{A}{\mu} \frac{\left(P_{b}-P_{a}\right)}{L}
$$

where $Q$ is the total discharge, $k$ is the permeability, $A$ is the area, $\left(P_{b}-P_{a}\right)$ is the pressure drop, and $L$ is the length over which the pressure drop. Darcy's law is an equation that describes the flow of a fluid in a porous medium [11].

$$
q=-\frac{k}{\mu} \nabla P
$$

where $q=\frac{Q}{A}$ is the flux or Darcy flux(discharge per unit area) and $\nabla P=\frac{\left(P_{b}-P_{a}\right)}{L}$ is pressure gradient vector.
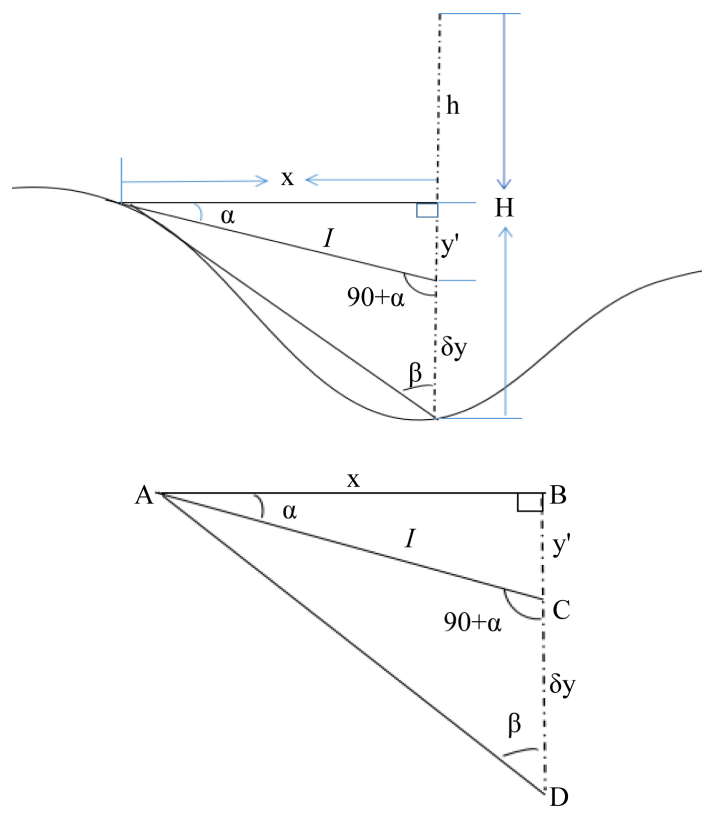

Figure 1. Contour region of a river bed [8]. 
We have an equation which shows relationship between the Darcy flux and the fluid velocity in the pore [12] as given below:

$$
\begin{gathered}
U=\frac{q}{\phi} \\
\Rightarrow q=U \phi
\end{gathered}
$$

substituting Equation (3) into Equation (2), we have:

$$
\begin{gathered}
U \phi=-\frac{k}{\mu} \nabla P \\
\Rightarrow \nabla P=-\frac{\mu \phi}{k} U
\end{gathered}
$$

$\nabla P$ gives the pressure variation in the channel and $U$ is the flow velocity.

\section{Model Analysis}

To solve for flow velocity $(U)$, we shall consider the equations governing the evolution of the weakly non-linear dispersive wave train in shallow water with varying nonuniform bottom topography [8].

$$
\begin{gathered}
\frac{\partial \eta}{\partial t}+\frac{\partial}{\partial x}[U(h+x \cot (\theta+\psi t))]=0 \\
\frac{\partial \eta}{\partial t}+U \frac{\partial U}{\partial x}+g \frac{\partial \eta}{\partial x}+\lambda \frac{\partial^{3} U}{\partial x^{3}}=\frac{\{h+x \cot (\theta+\psi t)\}^{2}}{3} \frac{\partial^{3} U}{\partial x^{2} \partial t}
\end{gathered}
$$

we consider the $x$ axis as the horizontal and normal to the shore line, the $z$ axis as the vertical such that $z=\eta(x, t)$ represents the wave profile occurrence on the water surface, $z=h$ is the constant water depth at the level bottom regions of the shallow water flow as measured from the undisturbed water level and $h+x \cot \beta$ is the flow depth over the contour region and $\beta=\theta+\psi t$.

We visited work by [2] [8] [10] [13] [14] to solve the problem. Though they used different methods in their analysis but Homotopy perturbation method (HPM) is applied here, to solve for $U(x, t)$. Then choosing initial conditions as:

$$
\begin{gathered}
\eta(x, 0)=\eta_{0}=x \cot \hat{\beta} \text { where } \hat{\beta}=\theta \text { at } t=0 \\
U(x, 0)=U_{0}=A \cos x
\end{gathered}
$$

We construct a homotopy as follow:

$$
\begin{gathered}
(1-S) \frac{\partial \eta}{\partial t}+S\left[\frac{\partial \eta}{\partial t}+\frac{\partial}{\partial x}(U[h+x \cot \beta])\right]=0 \\
(1-S) \frac{\partial U}{\partial t}+S\left[\frac{\partial U}{\partial t}+U \frac{\partial U}{\partial x}+g \frac{\partial \eta}{\partial x}+\lambda \frac{\partial^{3} U}{\partial x^{3}}-\frac{[h+x \cot \beta]^{2}}{3} \frac{\partial^{3} U}{\partial x^{2} \partial t}\right]=0
\end{gathered}
$$

where $S$ is an embedding parameter in $[0,1]$, if $S=0$, we have $\frac{\partial \eta}{\partial t}=0$ and $\frac{\partial U}{\partial t}=0$. But if $S=1$, we recover the original equation.

Expanding Equations (9) and (10), we have: 


$$
\begin{gathered}
\frac{\partial \eta}{\partial t}=-S\left[\frac{\partial \eta}{\partial t}+\frac{\partial}{\partial x}(U[h+x \cot \beta])-\frac{\partial \eta}{\partial t}\right] \\
\frac{\partial U}{\partial t}=-S\left[\frac{\partial U}{\partial t}+U \frac{\partial U}{\partial x}+g \frac{\partial \eta}{\partial x}+\lambda \frac{\partial^{3} U}{\partial x^{3}}-\frac{[h+x \cot \beta]^{2}}{3} \frac{\partial^{3} U}{\partial x^{2} \partial t}-\frac{\partial U}{\partial t}\right]
\end{gathered}
$$

The basic assumption is, the solution of Equations (5) and (6) with the initial conditions can be written as a power series in $S$ :

$$
\begin{gathered}
\eta=\eta_{0}+S \eta_{1}+S^{2} \eta_{2}+S^{3} \eta_{3}+\cdots \\
U=U_{0}+S U_{1}+S^{2} U_{2}+S^{3} U_{3}+\cdots
\end{gathered}
$$

Substituting Equations (13) and (14) into Equations (11) and (12), and expand. Also equating the terms with identical powers of $S$, and substituting initial conditions, but let $\theta=\hat{\beta} \Rightarrow$ at $t=0, \beta=\theta=\hat{\beta}$ for $\eta_{0}$, so we have:

$$
\begin{aligned}
& \eta_{0}(x, t)=x \cot \hat{\beta} \\
& U_{0}(x, t)=A \cos x \\
& \eta_{1}(x, t)=A \operatorname{th} \sin x+\frac{A}{\psi} x \sin x \ln [\sin \beta]-\frac{A}{\psi} \cos x \ln [\sin \beta] \\
& U_{1}(x, t)=\left[\frac{A^{2}}{2} \sin 2 x-g \cot \hat{\beta}-A \lambda \sin x\right] t \\
& \eta_{2}(x, t)=\left[\frac{A^{2}}{2} \sin 2 x-g \cot \hat{\beta}-A \lambda \sin x+A^{2} x \cos 2 x-A x \lambda \cos x\right] \\
& \times\left[\frac{1}{\psi} t \ln (\sin \beta)-\frac{\mathrm{i}}{2 \psi^{2}}\left[\beta^{2}-L i_{2}(-\mathrm{i} \sin 2 \beta-\cos 2 \beta+1)\right]\right. \\
&\left.-\frac{1}{\psi^{2}} \beta \ln (2 \sin \beta)-\frac{\beta}{\psi} \ln \left(\frac{\mathrm{i}}{2}\right)\right]+\left[A^{2} h \cos 2 x-A h \lambda \cos x\right] \frac{t^{2}}{2} \\
& U_{2}(x, t)= {\left[A^{3} \cos x \cos 2 x-A^{2} \lambda+\frac{A^{3}}{2} \sin x \sin 2 x-A g \sin x \cot \hat{\beta}-A g h \cos x\right.} \\
&+\left.4 A^{2} \lambda \cos 2 x-A \lambda^{2} \cos x \frac{t^{2}}{2}\right]+\frac{h^{2}}{3}\left[-2 A^{2} \sin 2 x+A \lambda \sin x\right] t \\
&-\frac{A}{\psi} g[2 \sin x+x \cos x]\left[\frac{i}{2 \psi}\left[\beta^{2}-L i_{2}(-\mathrm{i} \sin (2 \beta)-\cos (2 \beta)+1)\right]\right. \\
&+\left.\frac{1}{\psi} \beta \ln (2 \sin \beta)+\frac{\beta}{\psi} \ln \left(\frac{\mathrm{i}}{2}\right)\right]+\frac{1}{3}\left[-2 A^{2} \sin 2 x+A \lambda \sin x\right] \\
& \times {\left[\frac{2 h}{\psi} x \ln [\sin \beta]-x^{2}\left[\frac{1}{\psi} \cot \beta+t+\frac{\hat{\beta}}{\psi}\right]\right] } \\
& {[}
\end{aligned}
$$

Recalling Equations (13) and (14), we have:

$$
\begin{gathered}
\eta(x, t)=\eta_{0}(x, t)+S \eta_{1}(x, t)+S^{2} \eta_{2}(x, t)+S^{3} \eta_{3}(x, t)+\cdots \\
U(x, t)=U_{0}(x, t)+S U_{1}(x, t)+S^{2} U_{2}(x, t)+S^{3} U_{3}(x, t)+\cdots
\end{gathered}
$$

Let the $o\left(S^{3}\right)$ be very small, such that by asymptotic series approximation, 
we have:

$$
\begin{aligned}
& U(x, t)=U_{0}(x, t)+S U_{1}(x, t)+S^{2} U_{2}(x, t)+o\left(S^{3}\right) \\
& U(x, t)-U_{0}(x, t)-S U_{1}(x, t)-S^{2} U_{2}(x, t)<o\left(S^{3}\right) \\
& U(x, t) \approx U_{0}(x, t)+S U_{1}(x, t)+S^{2} U_{2}(x, t)
\end{aligned}
$$

Also, we have:

$$
\begin{aligned}
& \eta(x, t)=\eta_{0}(x, t)+S \eta_{1}(x, t)+S^{2} \eta_{2}(x, t)+o\left(S^{3}\right) \\
& \eta(x, t)-\eta_{0}(x, t)-S \eta_{1}(x, t)-S^{2} \eta_{2}(x, t)<o\left(S^{3}\right) \\
& \eta(x, t) \approx \eta_{0}(x, t)+S \eta_{1}(x, t)+S^{2} \eta_{2}(x, t)
\end{aligned}
$$

Substituting Equations (16), (18) and (20) into Equation (21) and evaluating at $S=1$, we have:

$$
\begin{aligned}
& U(x, t)=A \cos x+\left[\frac{A^{2}}{2} \sin 2 x-g \cot \hat{\beta}-A \lambda \sin x\right] t+\left[A^{3} \cos x \cos 2 x-A^{2} \lambda\right. \\
& \left.+\frac{A^{3}}{2} \sin x \sin 2 x-A g \sin x \cot \hat{\beta}-A g h \cos x+4 A^{2} \lambda \cos 2 x-A \lambda^{2} \cos x\right] \frac{t^{2}}{2} \\
& +\frac{h^{2}}{3}\left[-2 A^{2} \sin 2 x+A \lambda \sin x\right] t-\frac{A}{\psi} g[2 \sin x+x \cos x] \\
& \times\left[\frac{\mathrm{i}}{2 \psi}\left[\beta^{2}-L i_{2}(-\mathrm{i} \sin (2 \beta)-\cos (2 \beta)+1)\right]+\frac{1}{\psi} \beta \ln (2 \sin \beta)+\frac{\beta}{\psi} \ln \left(\frac{\mathrm{i}}{2}\right)\right] \\
& +\frac{1}{3}\left[-2 A^{2} \sin 2 x+A \sin x\right]\left[\frac{2 h}{\psi} x \ln (\sin \beta)-x^{2}\left[\frac{1}{\psi} \cot \beta+t+\frac{\hat{\beta}}{\psi}\right]\right]
\end{aligned}
$$

Also substituting Equations (15), (17) and (19) into Equation (22) and evaluating at $S=1$, we have:

$$
\begin{aligned}
\eta(x, t)= & x \cot \hat{\beta}+A \operatorname{th} \sin x+\frac{A}{\psi} x \sin x \ln [\sin \beta]-\frac{A}{\psi} \cos x \ln [\sin \beta] \\
& +\left[\frac{A^{2}}{2} \sin 2 x-g \cot \hat{\beta}-A \lambda \sin x+A^{2} x \cos 2 x-A x \lambda \cos x\right] \\
& \times\left[\frac{1}{\psi} t \ln (\sin \beta)-\frac{\mathrm{i}}{2 \psi^{2}}\left[\beta^{2}-L i_{2}(-\mathrm{i} \sin 2 \beta-\cos 2 \beta+1)\right]\right. \\
& \left.-\frac{1}{\psi^{2}} \beta \ln (2 \sin \beta)-\frac{\beta}{\psi} \ln \left(\frac{\mathrm{i}}{2}\right)\right]+\left[A^{2} h \cos 2 x-A h \lambda \cos x\right] \frac{t^{2}}{2}
\end{aligned}
$$

Recalling Equation (4), we have:

$$
\nabla P=-\frac{\mu \phi}{k} U
$$

where $\nabla P=\frac{\partial P}{\partial x}$ such that:

$$
\frac{\partial P(x, t)}{\partial x}=-\frac{\mu \phi}{k} U(x, t)
$$




$$
\Rightarrow P(x, t)=-\frac{\mu \phi}{k} \int U(x, t) \mathrm{d} x
$$

Substituting Equation (23) into Equation (25) and integrate w.r.t $x$, we have:

$$
\begin{aligned}
P(x, t)= & -\frac{\mu \phi}{k}\left[A \sin x+t\left[-\frac{A^{2}}{4} \cos 2 x-g x \cot \hat{\beta}-A \lambda \cos x\right]\right. \\
& +\frac{t^{2}}{2}\left[\frac{A^{3}}{3}\left[\frac{5}{2} \cos x \sin 2 x-2 \sin x \cos 2 x\right]-A^{2} \lambda x+A g \cos x \cot \hat{\beta}\right. \\
& \left.-A g h \sin x+2 A^{2} \lambda \sin 2 x-A \lambda^{2} \sin x\right]+\frac{h^{2}}{3} t\left[A^{2} \cos 2 x-A \lambda \cos x\right] \\
+ & \left.\frac{\beta}{\psi} \ln \left(\frac{\mathrm{i}}{2}\right)\right]\left[\frac{\mathrm{i}}{2 \psi}\left[\beta^{2}-L i_{2}(-\mathrm{i} \sin (2 \beta)-\cos (2 \beta)+1)\right]+\frac{1}{\psi} \beta \ln (2 \sin \beta)\right. \\
- & \frac{4 A^{2} h}{3 \psi} \ln (\sin \beta)\left[\frac{1}{4} \sin 2 x-\frac{x}{2} \cos 2 x\right] \\
- & \frac{A}{3} \lambda\left[\frac{1}{\psi} \cot \beta+t+\frac{\hat{\beta}}{\psi}\right]\left[-x^{2} \cos x+2 x \sin x+2 \cos x\right] \\
+ & \left.\frac{2 A^{2}}{3}\left[\frac{1}{\psi} \cot \beta+t+\frac{\hat{\beta}}{\psi}\right]\left[\frac{x}{2} \sin 2 x-\frac{x^{2}}{2} \cos 2 x+\frac{1}{4} \cos 2 x\right]\right]
\end{aligned}
$$

\section{Analysis of Result}

Having obtained the expression for the pressure gradient and the flow velocity in the contoured region along the channel, we assign value to the parameters, some of which we extract from literature and others are estimated to simulate the problem. Since we are dealing with real-life situations, we are considering only the real part of our solution. We have to note that the contour region is either filling or digging. This depends on the facts that $\beta=\theta+\psi t$ and that $0<\beta<\frac{\pi}{2}$, this is considered at different points of interest and is measured in radians such that we have:

\begin{tabular}{ccc}
\hline Parameters & Values & Source \\
\hline$A$ & 5 & Estimated \\
$\lambda$ & 0.1 & Estimated \\
$h$ & 6 & {$[8]$} \\
$\psi$ & 0.01 & {$[10]$} \\
$\phi$ & 0.5 & {$[3]$} \\
$k$ & $0.369,0.00223$ and 0.000084 & {$[15]$} \\
$\mu$ & 0.00089 & {$[16]$} \\
$g$ & 9.8 & Standard \\
\hline
\end{tabular}


$x$ is considered from -2 to 2 and is measured in meter $(\mathrm{m})$ while $t$ is measured in second(s). All the analysis carried out in this section is done using MAPLE 18. We considered the cases below:

For case 1, we studied the velocity at a particular time (fixed time) over different points of $x$. For this we fixed $t$ to $t=10$, that is 10 s of fluid flow over our area of interest (i.e. points of $x$ ). Different graphs of flow velocity were plotted for different values of $\theta$, this is shown in Figure 2.

We observed that at the entrance of the fluid into the contoured region there is a rise in flow velocity at the point $x=-2$ and as the fluid gets to the center of the region there is no velocity as the velocity is zero (i.e. where $x=0$ ) as shown in Figure 2. Also as the fluid continues coming into this region there is a build-up of velocity to get out of the region that is from the point after $x=0$ to $x=2$. We also observed that the deeper the contour, the higher the velocity, that is as $\theta$ decreased the region become deeper which also affects the flow velocity. This is the same as what happened in our shallow water flow in the real-life situation.

For case 2, we studied the velocity of the flow at a particular point over some time, say $t$ is from 0 s to $10 \mathrm{~s}$. We picked $x$ at the points $x=-2, x=2$ and $x=0$. For each of these points of $x$, different graphs of flow velocity were plotted for different values of $\theta$ as above.

It is observed that the velocity is higher at the highest angle at points $x=-2$ and $x=2$, but at the point, $x=0$ they all behave alike, this is because at that point there is no velocity. But at the center where $x=0$ there is turbulent, which resulted in that rise in velocity as shown in Figure 3(c).

Moreover, we simulate the flow velocity in 3-D for different flow height $(H)$ (i.e. different values of $\theta$ ).

Figure 4 agrees with what we have seen in the two-dimensional graph, that we have discussed above. We see that the deeper the contour region the higher the velocity compared to the other two as shown in Figure 4. This shows that the region contributes to the rate at which the fluid flow. We can say that as the

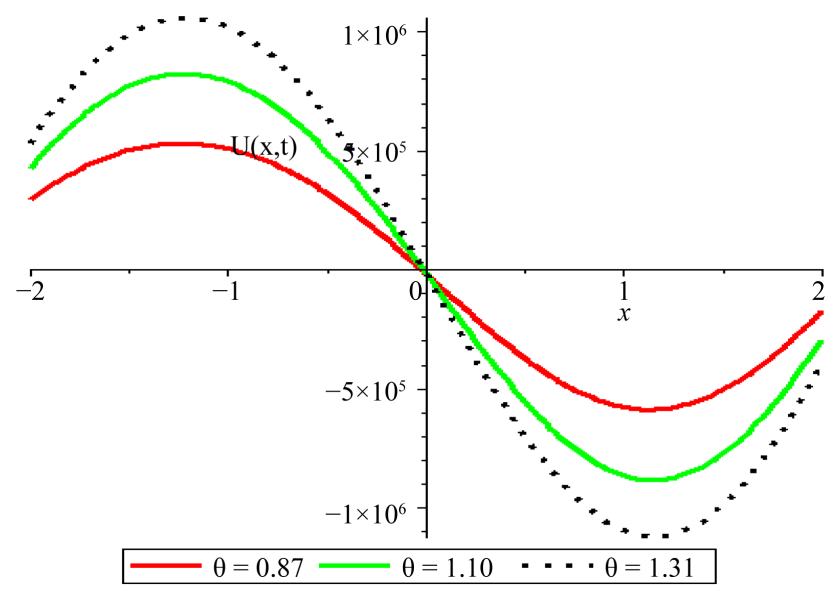

Figure 2. Effects of flow height $(H)$ on flow velocity over space $(x)$. 

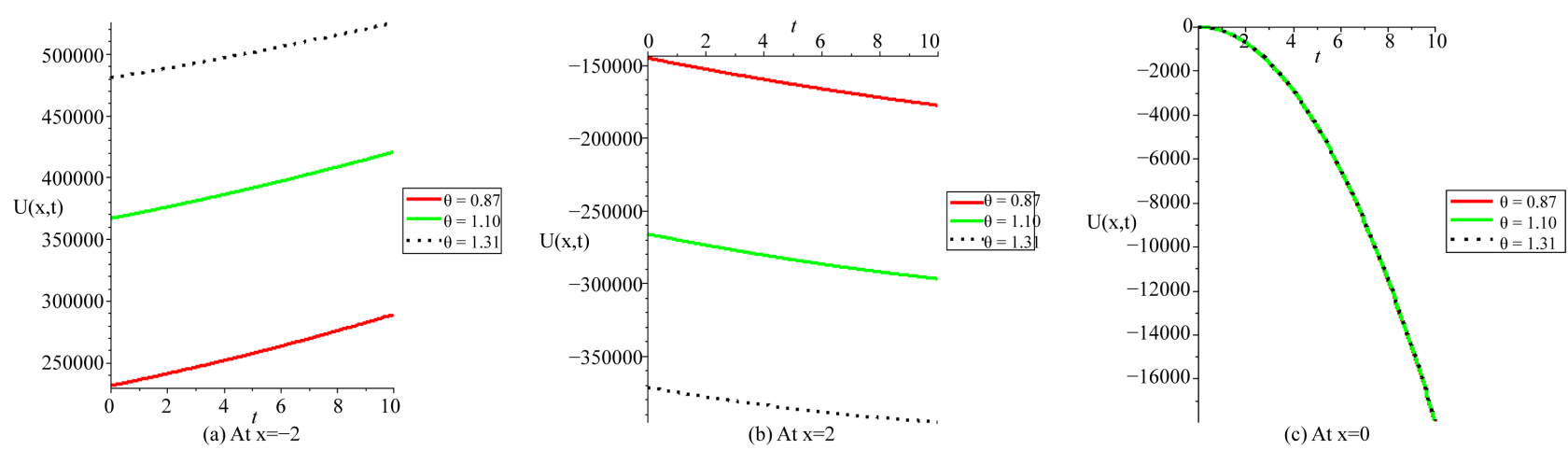

Figure 3. Effects of flow height $(H)$ on flow velocity over time $(t)$.
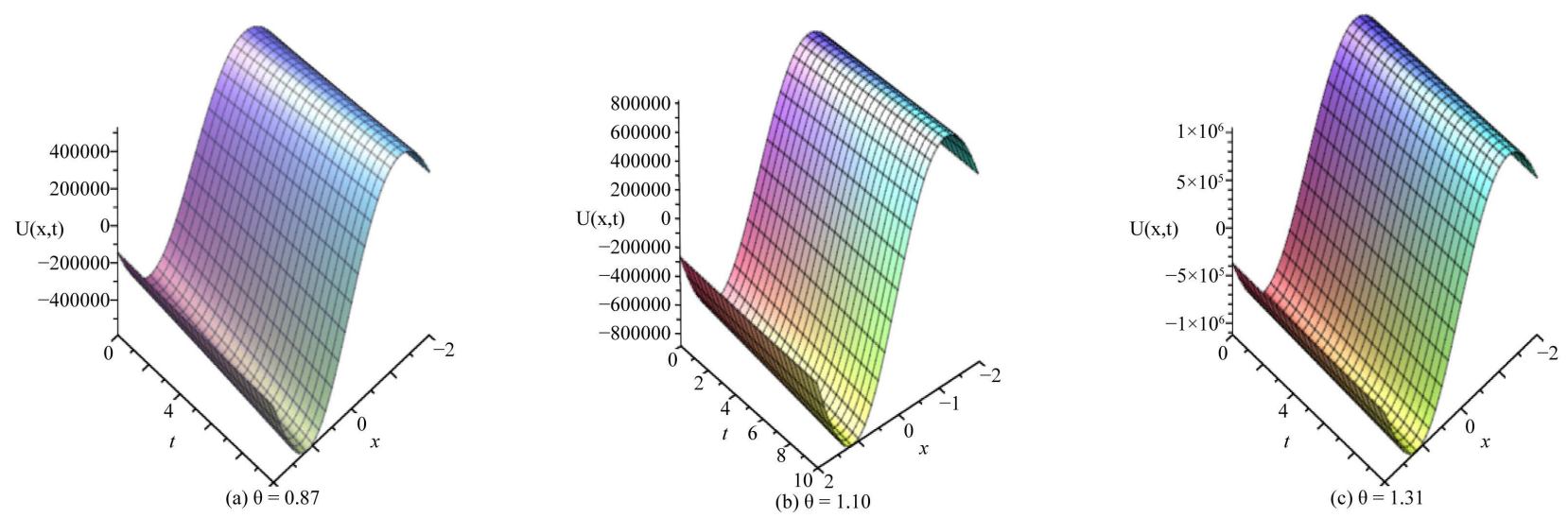

Figure 4. Effects of flow height $(H)$ on flow velocity.

contour region is being dug or filled, the velocity changes with respect to the rate at which it is dug or filled. But once the contour region is filled that is if there is no longer contour, the velocity remains unchanged.

More so, for case 3, since we derived our expression for pressure $(P)$ in terms of flow velocity $(U)$ and permeability $(k)$, we are studying the pressure drop, using different values of permeability $(k)$. At $k=0.369, k=0.00223$ and also $k=0.000084$ [15]. This is shown in Figure 5.

From Figure 5, we observed that when $k=0.000084$, there is a pressure build-up within the region under consideration and this is a result of the compactness of the bed material. This implies that at low permeability, there is going to be a rise in pressure. When $k=0.00223$, there are slight changes in the pressure along the channel. This is as a result of the arrangement of the bed materials. And lastly, when $k=0.369$, there are insignificant changes in the pressure, that is, there is loss of pressure due to the loss of water due to percolation in the channel. This shows that the bed materials arrangement contributes to the pressure gradient along the channel as shown in Figure 5.

We also looked at the effect of permeability on pressure over time, this is shown by Figure 6 .

We observed that there is rise in pressure $(P)$ over time $(t)$ at lowest value of permeability $(k)$ at different points of $x$ and as the permeability $(k)$ increase the 


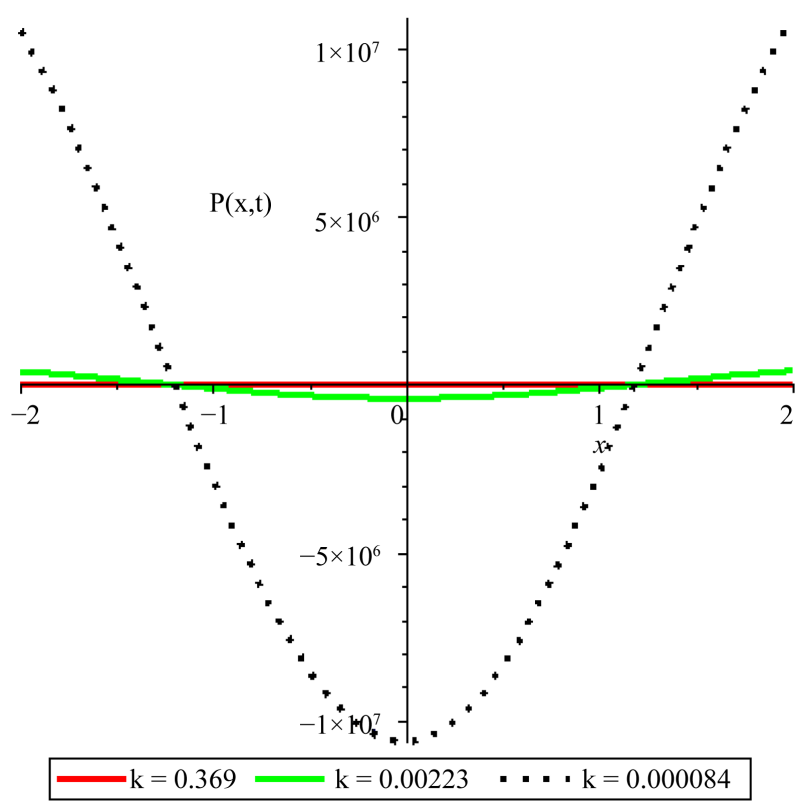

Figure 5. Effect of permeability on pressure over space.

pressure $(P)$ drop over time $(t)$. But at the highest value of permeability, there is an insignificant change in Pressure $(P)$ over time $(t)$ over different points of $x$ as shown in Figure 6. This shows that pressure will drop significantly when there is a change in permeability at each point of $x$.

And lastly, we fixed $k, x$ and varying time for different value of $\theta$, to check for effects of nonuniformity of the bed on the pressure along the channel. This we also graph as shown in Figure 7.

From Figure 7, we observed that when $\theta=1.31$, that is the deepest flow height $(H)$, the pressure $(P)$ is high and as the flow height $(H)$ is reduced the pressure $(P)$ also reduce, that is as the flow height $(H)$ increase the pressure $(P)$ increases too. And at a point into the contour region and out of the region, the pressure $(P)$ is zero and this is equidistant to the center sideways. At this point, the channel experience equal pressure $(P)$ for any flow height $(H)$ of the region.

From Figure 8(a) and Figure 8(c), it is observed that the pressure $(P)$ in these two-point increases with time and the highest flow height $(H)$ recorded the highest pressure $(P)$ at any given time which also buttress the point that the higher the contour the higher the pressure $(P)$. But in Figure 8(b) we observed that the pressure $(P)$ decreased into negative very well, this is as a result of fluid flowing into this point and accumulate before going out of the contoured region. That is, this negative value implies that there is pressure $(P)$ build-up at this point in the region.

This we also did for pressure $(P)$ in 3-D for permeability $(k)$ and we have:

Figure 9 also agreed with what we have in Figure 7 and Figure 8 as there is a rise in the pressure $(P)$ as the permeability $(k)$ decrease. As shown in Figure 9(a), we see clearly that, that insignificant change in pressure $(P)$ at $k=0.369$ in Figure 5 and Figure 6 does not mean it is not changing or that there is no 

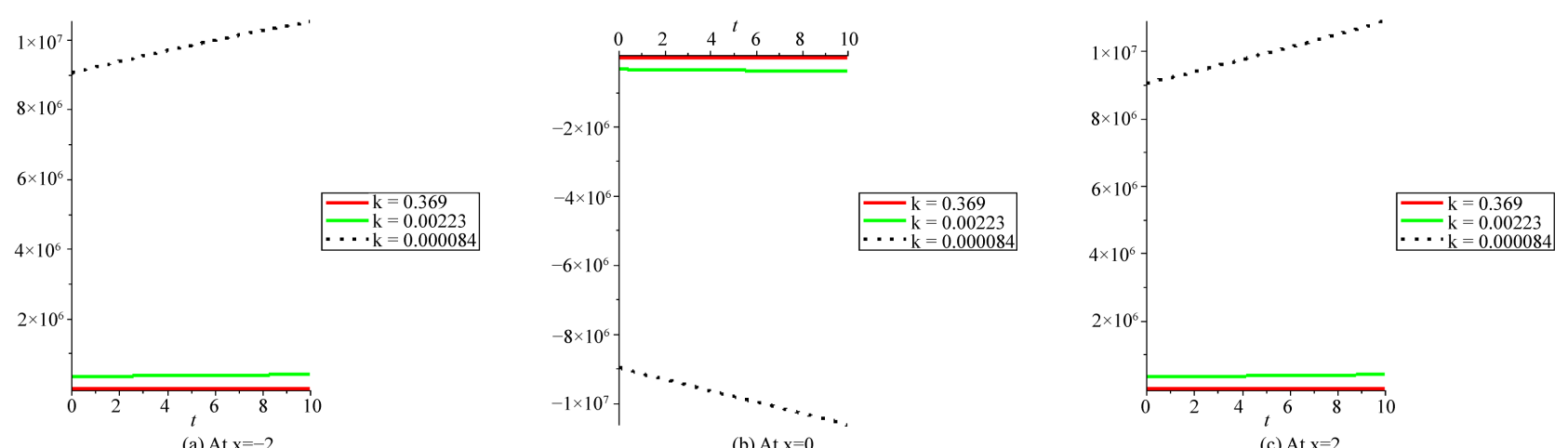

Figure 6. Effects of permeability on pressure over time $(t)$.

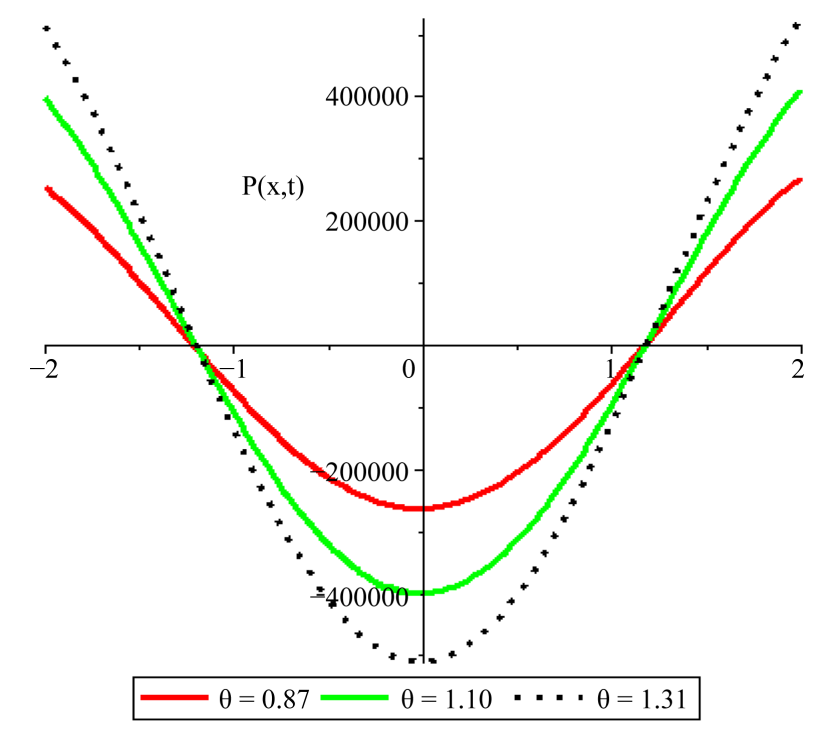

Figure 7. Effect of flow height $(H)$ on pressure over space.
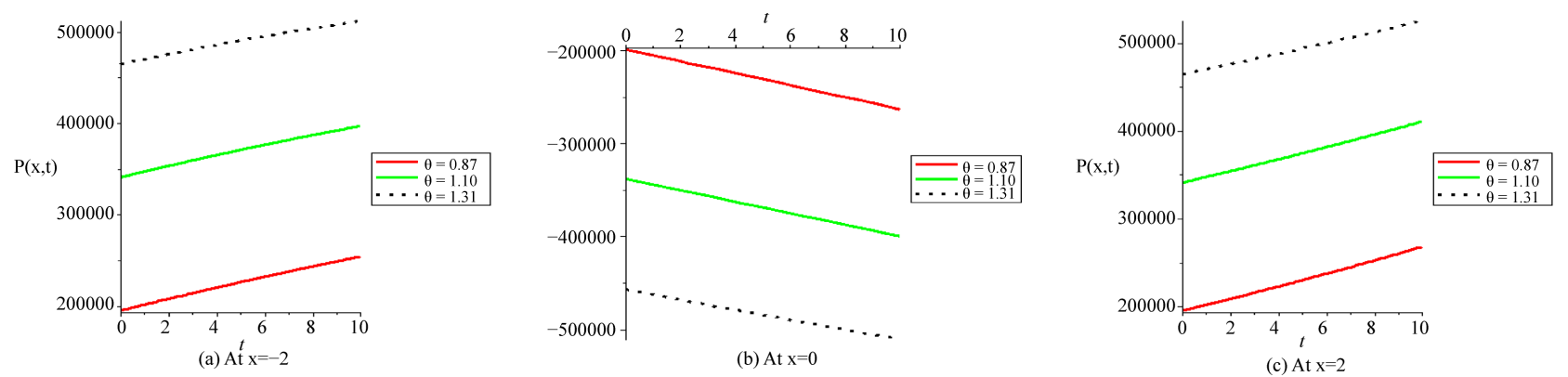

Figure 8. Effects of flow height $(H)$ on pressure $(P)$ over time $(t)$.

pressure $(P)$. It is just that the change is insignificant compared to the other two values of $k$. This is a result of a loosed packed bed of the channel which allowed fluid loss due to percolation. Also as shown in Figure 9(b), the pressure $(P)$ rise due to the fact that the packed bed allow little or no percolation, such that water is retained in the region which amount up to pressure $(P)$ build-up while Figure 9 (c) showed the facts that at $k=0.000084$, the packed bed is compactly packed 


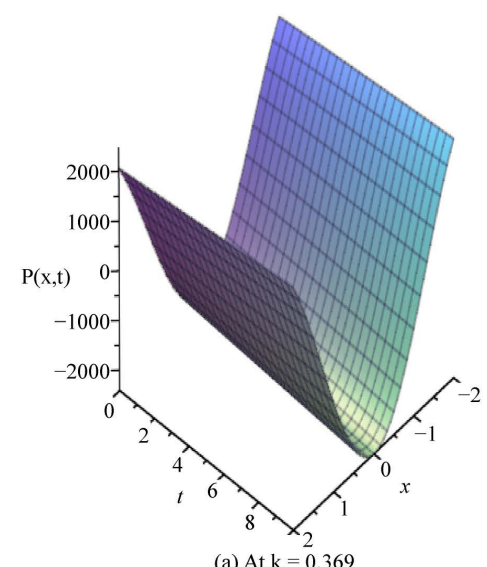

(a) At k $=0.369$

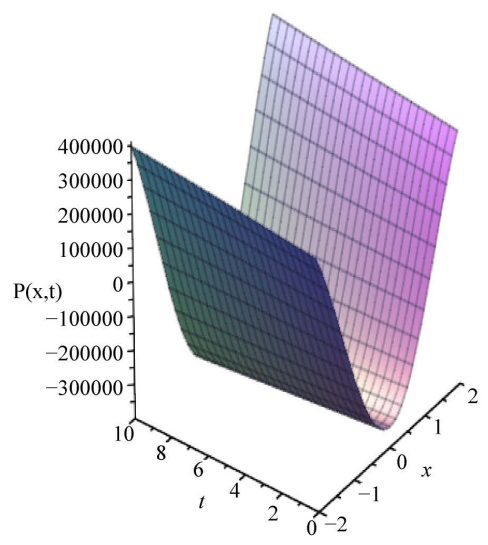

(b) At $\mathrm{k}=0.00223$

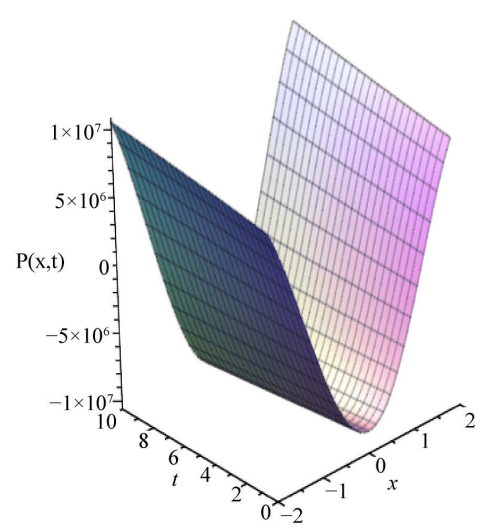

(c) At $\mathrm{k}=0.000084$

Figure 9. Effects of permeability on pressure.

together that it does not allow percolation at all, which may also mean there is a movement of fluid from the pores into the open channel since there is a great pressure $(P)$ build-up.

\section{Conclusions}

Based on the assumptions of the model, with regard to the conditions and with respect to the simulations above, we have that the deeper the contour, the faster the velocity but as the contour is filling, the velocity reduces and if the contour filled to form a level ground, then the velocity becomes uniform. This is what happens in real-life situations and it also agrees with [10]. Obviously, permeability $(k)$ of the bed materials has a great effect on the pressure $(P)$ along the channel. When the permeability $(k)$ is high, the pressure $(P)$ drops significantly due to the percolation of fluid through the larger size pores formed by the bed materials. This is what often causes some rivers to dry up at some season as much fluid is lost due to high permeability $(k)$. But when the permeability $(k)$ is low, the pressure $(P)$ rises in the channel because there is little or no fluid loss in the channel. Even in some cases, there is penetration of fluid from the pores into the channel due to capillarity from the pore. This is what we see in the water well at our homes. This also agrees with [3] and [4]. This is clearly seen in the desert, where the river beds are sandy. Drought is one major problem in these areas. The flow height $(H)$ also causes the pressure $(P)$ to either rise or fall. As fluid flows into the contoured region, there is pressure $(P)$ build-up in this region with respect to the height of this region, which is as predicted in the physical situation.

When the permeability of a river is high, there shall be less flooding in that area. Rainfall and snowmelt will cause temporary and gradual flooding. Impermeable, surfaces like roads, interlocks, sidewalks, and buildings contribute to urban runoff and flood. Because instead of water being absorbed into the ground and recharge the aquifer (underground water), it will not but causes a runoff. With these impermeable surfaces runoff during rainfall increases, causing rain- 
fall running directly into streams. This resulted in aquifer levels going down which also lower the level of the streams creating sudden, unseasonal flooding. Swamps and marshes should not be filled with dirt and should not be built upon as we are experiencing now. Without swamps and mashes which are also known as wetlands, rainwater flows directly into rivers and causes flooding when it overflows its bank. Dams or weirs may be built to store water or extracts energy. The management of the river should be taken seriously as rivers tend to undo the modifications made by people [3]. Agricultural activities should be monitored in the wetland area, as the use of machinery destroyed the permeability or made it less permeable or not permeable.

\section{Conflicts of Interest}

The authors declare no conflicts of interest regarding the publication of this paper.

\section{References}

[1] Craik, A.D. (2004) The Origins of Water Wave Theory. Annual Review of Fluid Mechanics, 36, 1-28. https://doi.org/10.1146/annurev.fluid.36.050802.122118

[2] Iornumbe, S. (2016) Stratification of Geophysical Flow. Ph.D. Dissertation, University of Nigeria, Nsukka.

[3] Mbah, G.C.E. and Udogu, C.I. (2015) Open Channel Flow Over a Permeable River Bed. Open Access Library Journal, 2, 1-7. https://doi.org/10.4236/oalib.1101475

[4] Udogu, C.I. (2013) Open Channel Flow over a Permeable River Bed. University of Nigeria, Nsukka.

[5] Okeke, E.O. (1999) On the Linearised Shallow Water Waves Over a Sloping Bottom. Nuovo Cimento della Societa Italiana di fisica C, No. 6, 72.

https://doi.org/10.1007/BF02511373

[6] Okeke, E.O. (1985) A Work on the Shallow Water Waves. Journal of the Nigerian Mathematical Society, 4, 89-93.

[7] Mbah, G.C.E. and Ezeorah, J.N. (2007) On the Wave Equations of Shallow Water with Non-Uniform Bottom Topography. Journal of Nigerian Association of Mathematical Physics, 12, 143-150.

[8] Mbah, G.C.E. (2008) Shallow Water Flow Over a Varying Non-Uniform Bottom Region. Proceedings of the First International Seminar on Theoretical Physics \& National Development, 1, 238-254.

[9] El, G.A., Grimshaw, R.H.J. and Smyth, N.F. (2009) Transcritical Shallow Water Flow Past Topography: Finite-Amplitude Theory. Journal of Fluid Mechanics, 640, 187-214. https://doi.org/10.1017/S0022112009991315

[10] Mbah, G.C.E. and Isienyi, S.U. (2018) Shallow Water Flow with Non-Uniform Bottom Topography. Journal of Nigerian Society of Mathematical Biology, 1, 67-93.

[11] Abbas, M.N. (2011) Modeling of Porosity Equation for Water Flow through Packed Bed of Monosize Spherical Packing. Journal of Engineering and Development, 15, 205-226.

[12] Bear, J. (2018) Modeling Phenomena of Flow and Transport in Porous Media. Springer International Publishing AG, Switzerland. https://doi.org/10.1007/978-3-319-72826-1 
[13] Peregrine, D.H. (1972) Equations for Water Waves and the Approximation behind them. Journal of Fluid Mechanics, 59, 95-121.

https://doi.org/10.1016/B978-0-12-493250-0.50007-2

[14] Whitham, G.B. (1974) Linear and Nonlinear Wave. John Wiley \& Sons Inc., Canada.

[15] Geotechdata.info (2013) Soil Void Ratio.

http://www.geotechdata.info/parameter/void-ratio.html

[16] Engineers Edge (2000) Water-Density Viscosity Specific Weight. https://www.engineersedge.com/physics/water density viscosity specific weight 13146.htm 